\title{
THE GENERAL CHAIN TRANSFORM AND SELF-RECIPROCAL FUNCTIONS
}

\section{CYRIL NASIM}

\author{
Department of Mathematics and Statistics \\ The University of Calgary \\ Calgary, Alberta T2N 1N4 Canada \\ (Received December 11, 1984)
}

ABSTRACT. A theory of a generalized form of the chain transforms of order $n$ is developed, and various properties of these are established including the Parseval relation. Most known cases of the standard theory are derived as special cases. Also a theory of self-reciprocal functions is given, based on these general chain transforms; and relations among various classes of self-reciprocal functions are established.

KEY WORDS AND PHRASES. Fourier transform, chain transforms, ic 2 lin transforms, Parseval theorem, kermel functions, $L^{2}$-space, self-reciprocal functions, gamma function, Riemann-leta function, Bessel functions, Whittaker functions.

1980 MATILIIATICS SUBJECT CLASSIFICATION CODL. $44 A 05$

1. INTRODUCTION.

The standard and the simplest definition of a function $g$ to be the integral transforms of $f$ with respect to $k$ is that

$$
g(x)=\int_{0}^{\infty} f(t) k(x t) d t
$$

where $k$ is called the kernal, [1,VIII]. If further

$$
f(x)=\int_{0}^{\infty} g(t) k(x t) d t,
$$

then we say that $f$ and $g$ are pair of $k$-transforms. Under less stringent conditions the equations (1.1) and (1.2) can be replaced by

$$
\begin{aligned}
& \qquad \int_{0}^{x} f(t) d t=\int_{0}^{\infty} t^{-1} f(t) k_{1}(x t) d t \\
& \qquad \int_{0}^{\infty} f(t) d t=\int_{0}^{\infty} t^{-1} g(t) k_{1}(x t) d t \\
& \text { where } k_{1}(x)=\int_{0}^{x} k(t) d t
\end{aligned}
$$

The above reciprocity formulae define the pair $f$ and $g$, generally known as Watson transforms. lany generalizations of this reciprocal relation have been given, and 
one of the directions was pointed out by Fox, [2], who introduced the idea of chain transforms. He showed that for chains of the form

$$
\begin{aligned}
& g_{2}(x)=\int_{0}^{\infty} g_{1}(u) k_{1}(u x) d u \\
& g_{n}(x)=\int_{0}^{\infty} g_{n-1}(u) k_{n-1}(u x) d u \\
& g_{1}(x)=\int_{0}^{\infty} g_{n}(u) k_{n}(u x) d u,
\end{aligned}
$$

there exists a theory similar to the standard theory for $n=2$, i.e., the Watson transformations. Also, Duggal, [3], considered the pair of equations.

$$
\begin{aligned}
& \int_{0}^{\infty} \frac{1}{x} V\left(\frac{u}{x}\right) f(x) d x=\int_{0}^{\infty} k(u x) g(x) d x \\
& \int_{0}^{\infty} \frac{1}{x} v\left(\frac{u}{x}\right) g(x) d x=\int_{0}^{\infty} k(u x) f(x) d x,
\end{aligned}
$$

using convolution of the functions $f$ and $g$ and characterizing the relationship between $f$ and $g$. If $V \equiv v$ are taken to be the Heaviside step functions, then (1.5) and (1.6) reduce to the equations similar to the equations (1.1) and (1.2) above, thus extending the Watson's definition.

In this paper we shall combine th- above mentioned two extensions of the usual Watson integral transformations. We shall give a generalized form of the chain transforms of order $n$ and develop properties parallel to those of the standard theory. Most of the well-known results are deduced as special cases. Further a theory of self-reciprocal functions is developed based on these general chain transforms; and again many standard results are deduced as special cases. In the end various examples are given to illustrate the general nature of the main theory developed and its special cases.

2. PRELIMINARY RESULTS.

Definition 2.1 Let $f \in L^{2}(0, \infty)$. Then define

$$
\begin{aligned}
& M[f(x) ; s] \equiv F(s)=\int_{0}^{\infty} f(x) x^{s-1} d x, \\
& s=\frac{1}{2}+i,-\infty<\tau<\infty . F(s) \text { is said to be the Mellin transform of } f(x) \text {; the }
\end{aligned}
$$
then

$$
M^{-1}[F(s) ; x] \equiv f(x)=\frac{1}{2 \pi i} \int_{\frac{1}{2}-i \infty}^{\frac{1}{2}+i_{\infty}^{\infty}} F(s) x^{-s} d s
$$

the integral existing in the mean-square sense; we say that $f(x)$ is the inverse Mellin transform of $F(s), s=\frac{1}{2}+i \tau,-\infty<\tau<\infty,[1, I]$.

The Parseval theorem, [1,III].

If $f$ and $g \in L^{2}(0, \infty)$ and have Mellin transforms $F(s)$ and $G(s)$ respectively, then

$$
\int_{0}^{\infty} f(x t) g(t) d t=\frac{1}{2 \pi i} \int_{\frac{1}{2}-i \infty}^{\frac{1}{2}+i \infty} F(s) G(1-s) x^{-s} d s
$$


Definition 2.2. A function $f$ is said to be self-reciprocal with respect to the kernel $k$ if $f$ is its own $k$-transform, satisfying the identity

$$
f(x)=\int_{0}^{\infty} f(t) k(x t) d t
$$

or its equivalent. We then say that

$$
f \in R_{k}
$$

Although most of the results hold for functions involved under less stringent conditions, but we shall mainly work in the Hilbert space $L^{2}$, for convenience and elegance of the theory of integral transforms in this setting.

3. CHAIN TRANSFORMS.

We consider the system of $n$ integral equations

$$
\int_{0}^{\infty} k_{i}(u x) f_{i+1}(x) d x=\int_{0}^{\infty} v_{i}(u x) \tilde{f}_{i}(x) d x
$$

where $i=1,2, \ldots, n$ and $\tilde{f}(x)=\frac{1}{x} f\left(\frac{1}{x}\right)$. For simplicity we shall assume that no two $f_{i}$ are equal, although it is not essential. We shall refer to the functions $k_{i}$ and $v_{i}$ as kernels. Now, we give one of our major results.

Theorem 3.1 .

If

(i) $k_{i}, v_{i}, f_{i}$ and $f_{n+1}$ a11 $\in L^{2}(0, \infty), i=1,2, \ldots, n$.

(ii) $\int_{0}^{\infty} k_{i}(u x) \tilde{f}_{i+1}(x) d x=\int_{0}^{\infty} v_{i}(u x) \tilde{f}_{i}(x) d x, i=1,2, \ldots, n$ where $\tilde{f}(x)=\frac{1}{x} f\left(\frac{1}{x}\right)$, and

$$
\text { (iii) } P(s)=\prod_{i=}^{n} \frac{V_{i}(s)}{K_{i}(s)} \in L^{\infty}\left(\frac{1}{2}-i \infty, \frac{1}{2}+i \infty\right) \text {, }
$$

then

$$
\int_{0}^{u} f_{n+1}(x) d x=\int_{0}^{\infty} x^{-1} p_{1}(u x) \tilde{f}_{1}(x) d x
$$

where $p_{1}(x)=\frac{1}{2 \pi i} \int_{\frac{1}{2}-i \infty}^{\frac{1}{2}+i \infty} \frac{P(s)}{1-s} x^{1-s} d s$,

and $V_{i}(s)$ and $K_{i}(s)$ denote the Mellin transforms of $v_{i}(x)$ and $k_{i}(x)$ respectively on $s=\frac{1}{2}+i \tau,-\infty<\tau<\infty$.

PROOF. Due to the hypothesis (i) and the fact that $f_{i} \in L^{2}(0, \infty)$ whenever $\tilde{f}_{i} \in L(0, \infty)$, the integrals in $(3.1)$ exist and are in fact absolutely convergent. Also, the $L^{2}$-functions in (i) have Mellin transforms which belong to $L^{2}\left(\frac{1}{2}-i \infty\right.$, $\left.\frac{1}{2}+i \infty\right)$ due to definition (2.1) above.

Now due to the Parseval theorem for Mellin transforms of $L^{2}$-functions, applied to both sides of (3.1), we have

$$
K_{i}(s) F_{i+1}(s)=V_{i}(s) F_{i}(s) \quad \text { a.e. }
$$

on $s=\frac{1}{2}+i \tau,-\infty<\tau<\infty, i=1,2, \ldots, n$. On successive elimination and using (3.2), we obtain 
Next we have

$$
\underset{n+1}{F}(s)=P(s) F_{1}(s)
$$

$$
f_{n+1}(x)=M^{-1}[\underset{n+1}{F}(s) ; x]
$$

therefore

$$
f_{n+1}(x)=\frac{1}{2 \pi i} \int_{\frac{1}{2}-i \infty}^{\frac{3}{2}+i \infty} P(s) F_{1}(s) x^{-s} d s,
$$

the integral converging in the mean-square sense, since $P\left(\frac{1}{2}+i \tau\right)$ is bounded and $F_{1}(s) \in L^{2}\left(\frac{1}{2}-i \infty, \frac{1}{2}+i \infty\right)$. Or from (3.6), we have

$$
\int_{0}^{u} f_{n+1}(x) d x=\frac{1}{2 \pi i} \int_{\frac{1}{2}-i \infty}^{\frac{3}{2}+i \infty} P(s) F_{1}(s) \frac{u^{-s}}{1-s} d s,
$$

the integration inside the integral sign is justified because of uniform convergence due to hypotheses (i) and (iii). Now since $P(s)$ is bounded, therefore $\frac{P(s)}{1-s} \epsilon$ $L^{2}\left(\frac{1}{2}-i \infty, \frac{1}{2}+i \infty\right)$ and hence

$$
x^{-1} p_{1}(x)=M^{-1}\left[\frac{P(s)}{1-s} ; x\right] \text {, }
$$

exists and $\epsilon L^{2}(0, \infty)$. Also

$$
\breve{f}_{1}(x)=M^{-1}\left[F_{1}(1-s) ; x\right],
$$

therefore due to the Parseval theorem applied to the right-hand side of (3.7), we obtain

$$
\int_{0}^{u} f_{n+1}(x) d x=\int_{0}^{\infty} x^{-1} p_{1}(u x) \tilde{f}_{1}(x) d x,
$$

as desired.

DEFINITION 3.1. The sequence of functions $\left\{f_{i}\right\}$ is said to be the general chain transform of order $n$, with respect to the kernels $k_{i}$ and $v_{i}, i=1,2, \ldots, n$, whenever the system (3.1) along with (3.3) is satisfied. It is now an easy matter, to prove a stronger result than that of Theorem 1, which we give below without proof.

THEOREM 3.2. Let the conditions of (i) and (ii) of THEOREM 1 hold. Then necessary and sufficient condition that (3.3) holds is that

$$
P(s)=\prod_{i=1}^{n} \frac{V_{i}(s)}{K_{i}(s)} \text {. }
$$

Next we shall give some special cases of the result of THEOREM 1 .

First let $n=1$.

COROLLARY 1. If

and

$$
\int_{0}^{\infty} k_{1}(u x) \widetilde{f}_{2}(x) d x=\int_{0}^{\infty} v_{1}(u x) \tilde{f}_{1}(x) d x
$$

then

$$
P(s)=\frac{V_{1}(s)}{K_{1}(s)} \text {, }
$$

$$
\int_{0}^{u} f_{2}(x) d x=\int_{0}^{\infty} x^{-1} p_{1}(u x) \hat{f}(x) d x,
$$

where $p_{1}(x)=\frac{1}{2 \pi i} \int_{\frac{1}{2}-i \infty}^{\frac{1}{2}+i \infty} \frac{P(s)}{1-s} x^{1-s} d s$. 
Thus if $k_{1}, v_{1}$ and $f_{1}$ are known, we have an inversion formula to retrive the unknown function $f_{2}$. If we further set

$$
v_{1}(x)=\left\{\begin{array}{l}
0 \text { if } 0<x<1, \\
\frac{1}{x} \text { if } x>1
\end{array}\right.
$$

we obtain a familiar unsymmetric transformation of Watson's type [1,VIII]; that is COROLLARY 2. If

$$
\int_{0}^{u} f_{1}(x) d x=\int_{0}^{\infty} \frac{u}{x} k_{1}\left(\frac{u}{x}\right) f_{2}(x) d x
$$

and

then

$$
P(s) K_{1}(s)=\frac{1}{1-s},
$$

$$
\int_{0}^{u} f_{2}(x) d x=\int_{0}^{\infty} p_{1}\left(\frac{u}{x}\right) f_{1}(x) d x
$$

Next let $n=2$. Then THEOREM 2, reduces to a known result [3], that is:

COROLLARY 3. If (i) $k_{i}, f_{i}$ and $f_{3} \in L^{2}(0, \infty), i=1,2$,

$$
\text { (ii) } \begin{aligned}
& \int_{0}^{\infty} k_{1}(u x) \mathfrak{f}_{2}(x) d x=\int_{0}^{\infty} v_{1}(u x) f_{1}(x) d x \\
& \int_{0}^{\infty} k_{2}(u x) \widetilde{f}_{3}(x) d x=\int_{0}^{\infty} v_{2}(u x) \widetilde{f}_{2}(x) d x
\end{aligned}
$$

and

$$
\text { (iii) } P(s)=\frac{V_{1}(s) V_{2}(s)}{K_{1}(s) K_{2}(s)} \in L^{\infty}\left(\frac{1}{2}-i \infty, \frac{1}{2}+i \infty\right)
$$

then

$$
\int_{0}^{u} f_{3}(x) d x=\int_{0}^{\infty} x^{-1} p_{1}(u x) \tilde{f}_{1}(x) d x
$$

where $p_{1}(x)=\frac{1}{2 \pi i} \int_{\frac{1}{2}-i \infty}^{\frac{1}{2}+i \infty} \frac{P(s)}{1-s} x^{1-s} d s$.

Suppose, now, $p_{1}(x)$ is differentiable, then from (3.4), we have

$$
p(x)=p_{1}^{\prime}(x)=\frac{1}{2 \pi i} \int_{\frac{3}{2}-i \infty}^{\frac{3}{2}+i \infty} P(s) x^{-s} d s .
$$

i.e. $\quad p(x)=M^{-1}[P(s) ; x]$.

Further let

$$
k_{i}(x)=\delta(1-x),
$$

$i=1,2, \ldots, n$ and $\delta$ being the Dirac delta function. Then the sequence (3.1), reduces to

$$
f_{i+1}(u)=\int_{0}^{\infty} v_{i}(u x) \tilde{f}_{i}(x) d x
$$

and consequently we have, a non-integrated version of Theorem 1:

$$
\begin{aligned}
& \text { THEOREM 3.3. If (i) } v_{i}, f_{i} \text { and } f_{n+1} \in L^{2}(0, \infty), i=1,2, \ldots, n \\
& \text { (ii) } f_{i+1}(u)=\int_{0}^{\infty} v_{i}(u x) \widetilde{f}_{i}(x) d x \\
& \text { (iii) } P(s)=\prod_{i=1}^{n} v_{i}(s),
\end{aligned}
$$


then

$$
\begin{array}{ll} 
& f_{n+1}(u)=\int_{0}^{\infty} p(u x) \widetilde{f}_{1}(x) d x, \\
\text { where } & p(x)=\frac{1}{2 \pi i} \int_{\frac{1}{2}-i \infty}^{\frac{3}{2}+i_{\infty}} P(s) x^{-s} d s .
\end{array}
$$

Letting $p(x)=\delta(1-x)$, and hence, its Mellin transform, $P(s)=1$ the above result, then, defines the chain transforms introduced by Fox. Note that all the above mentioned results are specializations of our Theorem 1 , showing the general nature of that result. Next we shall deduce a Perseval type relation for the general chain transforms as defined by Definition 3 . Let sequences $\left\{f_{i}\right\}$ and $\left\{g_{i}\right\}$ be chain transforms of order $n$ with respect to the kernels $k_{i}$ and $v_{i}$. Now consider

$$
\int_{0}^{\infty} x^{-1} f_{n+1}^{*}(u x) \tilde{g}_{1}(x) d x
$$

where

$$
f_{n+1}^{*}(u)=\int_{0}^{u} f_{n+1}(x) d x .
$$

By virtue of THEOREM 1 and the consequence (3.3), we have

Hence

$$
\begin{aligned}
\int_{0}^{\infty} x^{-1} f_{n+1}^{\star}(u x) \tilde{g}_{1}(x) d x & =\int_{0}^{\infty} x^{-1} \tilde{g}_{1}(x) d x \int_{0}^{\infty} t^{-1} p_{1}(u x t) \widetilde{f}_{1}(t) d t \\
& =\int_{0}^{\infty} t^{-1} \widetilde{f}_{1}(t) d t \int_{0}^{\infty} x^{-1} p_{1}(u x t) \tilde{g}_{1}(x) d x \\
& =\int_{0}^{\infty} t^{-1} \widetilde{f}_{1}(t) g_{n+1}^{*}(u t) d t \\
\int_{0}^{\infty} x^{-1} f_{n+1}^{*}(u x) \tilde{g}_{1}(x) d x & =\int_{0}^{\infty} t^{-1 \widetilde{f}_{1}}(t) g_{n+1}^{*}(u t) d t,
\end{aligned}
$$

establishing, formally, the Parseval relation. Further on differentiating both sides, formally, we have

$$
\int_{0}^{\infty} f_{n+1}(u x) \widetilde{g}_{1}(x)=\int_{0}^{\infty} \widetilde{f}_{1}(t) \widetilde{g}_{n+1}(u t) d t .
$$

Letting $n=1$, reduces the above to the usual Parseval Theorem, [1, III].

It is now an easy matter to develop an inversion theory for the general chain transforms. For instance if we assume that

$$
P(s) P(1-s)=1 \text {, }
$$

then from (3.3), due to the standard inversion formula, we have

$$
\int_{0}^{u} f_{1}(x) d x=\int_{0}^{\infty} x^{-1} p_{1}(u x) f_{n+1}(x) d x .
$$

Thus the unknown function $f_{1}$ is retrieved. On the other hand if we assume that

$$
\frac{1}{P(\theta)} \in E_{0}, \quad \theta \equiv-x \frac{d}{d x} \text {, }
$$

$E_{0}$, being the Lagunerre-Polya class [4, VII], then due to the known inversion techniques, [5]

$$
\frac{1}{P(\theta)}\left[f_{n+1}(x)\right]=f_{1}(x) \text {, }
$$

giving us the function $f_{1}$. 


\section{SELF-RECIPROCAL FUNCTIONS.}

Suppose $\left\{f_{i}\right\}$ is a sequence of general chain transforms of order $n$, with respeit to the kernels $k_{i}$ and $v_{i}$. If we then set $f_{n+1}(x)=f_{1}(x)$, in Theorem 1 , we have

THEOREM 4.1 . If (i) $k_{i}, v_{i}, f_{i} \in L^{2}(0, \infty), i=1,2, \ldots, n$,

(ii) $\int_{0}^{\infty} k_{i}(u x) \widetilde{f}_{i+1}(x) d x=\int_{0}^{\infty} v_{i}(u x) \widetilde{f}_{i}(x) d x$

$i=1,2, \ldots, n-1, \widetilde{f}(x)=\frac{1}{x} f\left(\frac{1}{x}\right)$,

$\int_{0}^{\infty} k_{n}(u x) f_{1}(x) d x=\int_{0}^{\infty} v_{n}(u x) \widetilde{f}_{n}(x) d x$

and

(iii) $P(s)=\prod_{i=1}^{n} \frac{V_{i}(s)}{K_{i}(s)} \in L^{\infty}\left(\frac{1}{2}-i \infty, \frac{1}{2}+i \infty\right)$,

then

$$
\begin{aligned}
& \int_{0}^{u} \tilde{f}_{1}(x) d x=\int_{0}^{\infty} x^{-1} p_{1}(u x) \widetilde{f}_{1}(x) d x, \\
& p_{1}(x)=\frac{1}{2 \pi i} \int_{\frac{1}{2}-i \infty}^{\frac{3}{2}+i \infty} \frac{P(s)}{1-s} x^{1-s} d s .
\end{aligned}
$$

From the conclusion (4.3), it follows that $f_{1}$ is self-reciprocal with respect to the kernel $p_{1}(x)$, in the sense of definition 2.2. Symbolically we say that

$$
\widetilde{f}_{1} \in R_{p_{1}}
$$

The particular case when $n=2$ is of special interest. In this case, many useful properties of self-reciprocal functions are established, including a procedure for generating self-reciprocal functions with respect to a given kernel. Now let $n=2$. Then,

$$
\begin{aligned}
& \text { COROLLARY 1. If (i) } k_{i}, v_{i} \text { and } f_{i} \in L^{2}(0, \infty), i=1,2 \text {, } \\
& \text { (ii) } \int_{0}^{\infty} k_{1}(u x) \breve{f}_{2}(x) d x=\int_{0}^{\infty} v_{1}(u x) \widetilde{f}_{1}(x) d x \\
& \int_{0}^{\infty} k_{2}(u x) f_{1}(x) d x=\int_{0}^{\infty} v_{2}(u x) f_{2}^{x}(x) d x \\
& \text { and } \\
& \text { (iii) } P(s)=\frac{V_{1}(s) V_{2}(s)}{K_{1}(s) K_{2}(s)}, \in L^{\infty}\left(\frac{1}{2}-i \infty, \frac{1}{2}+i \infty\right) \\
& \text { then } \\
& \widetilde{f}_{1} \in R_{p_{1}} \\
& \text { where } p_{1}(x)=\frac{1}{2 \pi i} \int_{\frac{1}{2}-i \infty}^{\frac{1}{2}+i \infty} \frac{P(s)}{1-s} x^{1-s} d s \text {. }
\end{aligned}
$$

PROOF. By the Parseval theorem for Mellin transforms, equation (4.4) and (4.5) give respectively,

$$
\begin{aligned}
& K_{1}(s) F_{2}(s)=V_{1}(s) F_{1}(s) \\
& K_{2}(s) F_{1}(1-s)=V_{2}(s) F_{2}(s),
\end{aligned}
$$

a.e. on $s=\frac{1}{2}+i \tau,-\infty<\tau<\infty$, involving the Mellin transform of the respective functions. Next define functions

and

$$
L(s)=\frac{V_{2}(1-s)}{K_{2}(1-s)} \quad L^{\infty}\left(\frac{1}{2}-i^{\infty}, \frac{1}{2}+i^{\infty}\right)
$$

$$
M(s)=\frac{V_{1}(s)}{K_{1}(s)} L(s), \quad s=\frac{1}{2}+i \tau,-\infty<\tau<\infty \text {. }
$$


Then from (4.6) and (4.7), we obtain, a.e. on $s=\frac{1}{2}+i \tau,-\infty<\tau<\infty$,

$$
F_{2}(s)=M(s) F_{2}(1-s)
$$

and

$$
F_{1}(s)=L(s) F_{2}(1-s) \text {. }
$$

A1so,

$$
P(s)=\frac{L(1-s)}{L(s)} M(s) .
$$

By the Parseval theorem for Mellin transform of $L^{2}$-functions (4.8) and (4.9) give, respectively,

$$
\begin{aligned}
& \int_{0}^{u} f_{2}(x) d x=\int_{0}^{\infty} x^{-1} m(u x) f_{2}(x) d x \\
& \int_{0}^{u} f_{1}(x) d x=\int_{0}^{\infty} x^{-1} \ell_{1}(u x) f_{2}(x) d x
\end{aligned}
$$

where

and

$$
m_{1}(x)=\frac{1}{2 \pi i} \int_{\frac{1}{2}-i_{\infty}^{\infty}}^{\frac{7}{2}+i_{\infty}^{\infty}} \frac{M(s)}{1-s} x^{1-s} d x .
$$

$$
\ell_{1}(x)=\frac{1}{2 \pi i} \int_{\frac{1}{2}-i \infty}^{\frac{1}{2}+i \infty} \frac{L(s)}{1-s} x^{1-s} d s .
$$

The functions $m_{1}$ and $\ell_{1}$ are defined by the integrals which exist in the mean-square sense, since both $M(s)$ and $L(s)$ are bounded on $s=\frac{1}{2}+i \pi,-\infty<\tau<\infty$, due to the hypothesis (iii) of Corollary 1 above, and consequently $\frac{M(s)}{1-s}$ and $\frac{L(s)}{1-s}$ both $\epsilon L^{2}\left(\frac{1}{2}-i \infty, \frac{1}{2}+i \infty\right)$. Thus, combining the above results, we have,

Corollary 2. If

$$
\begin{aligned}
& \text { (i) } f_{1} \text { and } f_{2} \in L^{2}(0, \infty), \\
& \text { (ii) } \int_{0}^{u} f_{2}(x) d x=\int_{0}^{\infty} x^{-1} m_{1}(u x) f_{2}(x) d x \\
& \text { (iii) } \int_{0}^{u} f_{1}(x) d x=\int_{0}^{\infty} x^{-1} \ell_{1}(u x) f_{2}(x) d x \\
& \text { (iv) } P(s)=\frac{L(1-s)}{L(s)} M(s)
\end{aligned}
$$

then

$$
f_{1} \in R_{p_{1}}
$$

where $\ell_{1}, m_{1}$ and $p_{1}$ are defined above.

This result gives us a procedure for generating a new class of self-reciprocal functions, given a self-reciprocal function and the kernels. Note that the hypothesis (ii) shows that $f_{2} \in R_{m_{1}}$. If all the functions involved are differentiable, we obtain a simpler form of the Corollary 2 above, that is:

Corollary 3. If (i) $f_{1}$ and $f_{2} \in L^{2}(0, \infty)$

and

$$
\begin{aligned}
& \text { (ii) } f_{2}(u)=\int_{0}^{\infty} m(u x) f_{2}(x) d x \text {, (i.e. } f_{2} \in R_{m} \text { ), } \\
& \text { (iii) } f_{1}(u)=\int_{0}^{\infty} \ell(u x) f_{2}(x) d x
\end{aligned}
$$

$$
\text { (iv) } P(s)=\frac{L(1-s)}{L(s)} M(s)
$$


then

For example, let

$$
\left.\tilde{f}_{1}(u)=\int_{0}^{\infty} p(u x) \ddot{f}_{1}(x) d x \text {, (i.e. } \tilde{f}_{1} \in R_{p}\right) \text {. }
$$

$$
m(x)=-2 \pi\left[\sin \frac{1}{2} \pi \nu J_{\nu}\left(4 \pi x^{\frac{1}{2}}\right)+\cos \frac{1}{2} \pi \nu\left\{Y_{\nu}\left(4 \pi x^{\frac{2}{2}}\right)-\frac{2}{\pi} K \nu\left(4 \pi x^{\frac{3}{2}}\right)\right\}\right], \nu \geq 0
$$

Now

$$
M(s)-\frac{\zeta\left(1-s-\frac{1}{2} \nu\right) \zeta\left(1-s+\frac{1}{2} \nu\right)}{\zeta\left(s-\frac{1}{2} \nu\right) \zeta\left(s+\frac{1}{2} \nu\right)} \text {, }
$$

where $\zeta(z)=\sum_{n=1}^{\infty} n^{-z}, R(z)>1$, the Riemann-Zeta function. Also,

$$
M(s)=\frac{(2 \pi)^{1-2 s} \Gamma\left(s+\frac{1}{2} \nu\right) \cos \frac{1}{2} \pi\left(s+\frac{1}{2} \nu\right)}{\Gamma\left(1-s+\frac{1}{2} \nu\right) \sin \frac{1}{2} \pi\left(s-\frac{1}{2} \nu\right)},
$$

by making use of the identity

$$
\zeta(s)=\pi^{s-1} 2^{s} \Gamma(1-s) \sin \frac{1}{2} \pi s \zeta(1-s) .
$$

It is an easy matter to see that

$$
M(s) M(1-s)=1 \text {, }
$$

hence $m(x)$ is a Fourier-Watson kernel. Further let

then

$$
L(s)=\operatorname{cosec} \frac{\pi}{2}\left(s-\frac{1}{2} \nu\right),
$$

$$
\begin{aligned}
P(s) & =\frac{L(1-s)}{L(s)} M(s) \\
& =\frac{(2 \pi)^{1-2 s} \Gamma\left(s+\frac{1}{2} v\right)}{\Gamma\left(1-s+\frac{1}{2} \nu\right)}, \\
\ell(x) & =H^{-1}[L(s) ; x]=\frac{2}{\pi} \frac{x^{-\frac{3}{2} \nu}}{1+x^{2}} ; \nu \geq 0,-\frac{1}{2} \nu<\operatorname{Re} s<2+\frac{1}{2} \nu
\end{aligned}
$$

and

$$
p(x)=M^{-1}[P(s) ; x]=2 \pi J_{\nu}\left(4 \pi x^{\frac{1}{2}}\right),-\frac{1}{2}<\operatorname{Re} s<\frac{3}{2} \text {. }
$$

Thus,

Corollary 4. If (i) $f \in R_{m}, m$ defined by (4.10),

$$
\begin{aligned}
& \text { (ii) } g(x)=\int_{0}^{\infty} \ell(x t) f(t) d t, \\
& \text { where } \ell(x)=\frac{2}{\pi} \frac{x^{-\frac{3}{2} \nu}}{1+x^{2}},
\end{aligned}
$$

then $\quad \tilde{g}(x) \in R_{p}$, where $p(x)=2 \pi J_{v}\left(4 \pi x^{\frac{1}{2}}\right)$.

In particular, let

$$
f(x)=x^{\frac{1}{2} \nu} \cos (2 \pi x)
$$

One can show that $f \in R_{m}$. Then

$$
\begin{aligned}
g(x) & =\int_{0}^{\infty} \ell(x t) f(t) d t \\
& =\frac{2}{\pi} x^{-\frac{1}{2} \nu} \int_{0}^{\infty} \frac{\cos (2 \pi t)}{1+x^{2} t^{2}} d t \\
& =x^{-\left(1+\frac{3}{2} \pi\right)} e^{-2 \pi / x}, \quad[6 ; 221(55)] .
\end{aligned}
$$

Now

$$
\int_{0}^{\infty} p(x t) \ddot{g}(t) d t=2 \pi \int_{0}^{\infty} t^{\frac{3}{2} \nu} e^{-2 \pi t} J_{\nu}\left(4 \pi x^{\frac{1}{2}} t^{\frac{3}{2}}\right) d t
$$




$$
\begin{aligned}
& =x^{\frac{3}{2} \nu} e^{-2 \pi x}, \quad[6 ; 29(10)] . \\
& =\tilde{g}(x),
\end{aligned}
$$

as predicted, thus verifying the result of Corollary 4. Next we shall give a slightly different version of Theorem 4.1, so that the function $f_{1}$, rather than the function $f_{1}$ is involved in the conclusion.

THEOREM 4.2. Let the conditions (i) and (ii) of Theorem 4.1 hold. Further if

$$
Q(s)=\prod_{i=1}^{n} \frac{V_{i}(1-s)}{K_{i}(1-s)} \in L^{\infty}\left(\frac{1}{2}-i \infty, \frac{1}{2}+i \infty\right)
$$

then

$$
\int_{0}^{\mu} f_{1}(x) d x=\int_{0}^{\infty} x^{-1} q_{1}(u x) f_{1}(x) d x, \quad\left(\text { or } f \in R_{q_{1}}\right. \text { ) }
$$

where

$$
q_{1}(x)=\frac{1}{2 \pi i} \int_{\frac{1}{2}-i \infty}^{\frac{1}{2}+i \infty} \frac{Q(s)}{1-s} x^{1-s} d s \text {. }
$$

Again the special case when $n=2$, is interesting, since it gives us a procedure for generating self-reciprocal functions.

$$
\begin{aligned}
& \text { COROLLARY 5. If (i) } f_{i}, v_{i} \text { and } k_{i} \quad L(0,), i=1,2, \\
& \text { (ii) } \int_{0}^{\infty} k_{1}(u x) \hat{f}_{2}(x) d x=\int_{0}^{\infty} v_{1}(u x) \tilde{f}_{1}(x) d x \\
& \int_{0}^{\infty} k_{2}(u x) \tilde{f}_{1}(x) d x=\int_{0}^{\infty} v_{2}(u x) \tilde{f}_{2}(x) d x \\
& \text { (iii) } Q(s)=\frac{V_{1}(1-s) V_{2}(1-s)}{K_{1}(1-s) K_{2}(1-s)},
\end{aligned}
$$

then

$$
\begin{aligned}
& \int_{0}^{u} f_{1}(x) d x=\int_{0}^{\infty} x^{-1} q_{1}(u x) f_{1}(x) d x \\
& \text { i.e. } f_{1} \in R_{q_{1}} .
\end{aligned}
$$

Now from the hypothesis (ii), by using the Parseval theorem for Mellin transforms, the two equations imply respectively,

$$
\begin{aligned}
& K_{1}(s) F_{2}(s)=V_{1}(s) F_{1}(s) \\
& K_{2}(s) F_{1}(1-s)=V_{2}(s) F_{2}(s),
\end{aligned}
$$

a.e., where $s=\frac{1}{2}+\tau,-\infty<\tau<\infty$.

If we consider the functions $L(s)$ and $M(s)$, defined earlier, then as before, we obtain

$$
F_{2}(s)=M(s) F_{2}(1-s)
$$

and

$$
F_{1}(s)=L(s) F_{2}(1-s), \quad \text { a.e. }
$$

The last two equations, then give, respectively,

and

$$
\int_{0}^{u} f_{2}(x) d x=\int_{0}^{\infty} x^{-1} m_{1}(u x) f_{2}(x) d x
$$

$$
\int_{0}^{u} f_{1}(x) d x=\int_{0}^{\infty} x^{-1} \ell(u x) f_{2}(x) d x
$$

where

$$
\ell_{1}(x)=\frac{1}{2 \pi i} \int_{\frac{1}{2}-i \infty}^{\frac{1}{2}+i \infty} \frac{L(s)}{1-s} x^{1-s} d s
$$


and

$$
m_{1}(x)=\frac{1}{2 \pi i} \int_{\frac{1}{2}-i \infty}^{\frac{1}{2}+i \infty} \frac{M(s)}{1-s} x^{1-s} d s .
$$

Thus we, formally, have

$$
\begin{aligned}
& \text { COROLLARY 6. If (i) } f_{1} \text { and } f_{2} \in L^{2}(0, \infty), \\
& \text { (ii) }(4.11) \text { and }(4.12) \text { are satisfied, } \\
& \text { (iii) } Q(s)=\frac{L(s)}{L(1-s)} M(1-s),
\end{aligned}
$$

then

$$
\int_{0}^{\mu} f_{1}(x) d x=\int_{0}^{\infty} x^{-1} q_{1}(u x) f_{1}(x) d x \text { where } \ell_{1}, m_{1}, q_{1} \text { are as def ined above. }
$$

It is not difficult to prove the above result rigorously, the functions $f_{1}$ and $f_{2} \epsilon$ $L^{2}(0, \infty)$ and $M(s), L(s)$ and $Q(s)$ are bounded on $s=\frac{1}{2}+i \tau,-\infty<\tau<\infty$, hence the Parseval theorem can be applied to give us the desired result. A non-integrated form of the above result is as follows:

$$
\begin{aligned}
\text { COROLLARY 7. If } & \text { (i) } f_{2}(u)=\int_{0}^{\infty} m(u x) f_{2}(x) d x \\
\text { (ii) } f_{1}(u) & =\int_{0}^{\infty} \ell(u x) f_{2}(x) d x
\end{aligned}
$$

then

The kernel

$$
f_{1}(u)=\int_{0}^{\infty} q(u x) f_{1}(x) d x
$$

$$
q_{1}\left(u^{2}\right)=\int_{0}^{u} q(x) d x
$$

and the kernels $\ell(x)$ and $m(x)$ are defined similarly. Note that the above results gives us a procedure for generating self-reciprocal functions. For example, let

$$
m(x)=x^{\frac{3}{2}} J_{v}(x),
$$

then its Mellin transform is given by $[7 ; 326(1)]$

Now, let

$$
M(s)=2^{s-\frac{1}{2}} \frac{\Gamma\left(\frac{1}{2} \nu+\frac{1}{2} s+\frac{1}{4}\right)}{\Gamma\left(\frac{1}{2} \nu-\frac{1}{2} s+\frac{3}{4}\right)},
$$

$$
\ell(x)=(x / 2)^{\frac{1}{2}(\mu+v+1)} K_{ \pm \frac{1}{2}(\nu-\mu)}(x),
$$

$K_{\eta}$ being the usual Bessel function of third kind, and its Mellin transform is Therefore

$$
L(s)=2^{s-\frac{1}{2}} \Gamma\left(\frac{1}{2} \nu+\frac{1}{2} s+\frac{1}{4}\right)\left(\frac{1}{2} \mu+\frac{1}{2} s+\frac{1}{4}\right) .
$$

$$
\begin{aligned}
Q(s) & =\frac{L(s)}{L(1-s)} M(1-s) \\
& =2^{s-\frac{1}{2}} \frac{\Gamma\left(\frac{1}{2} \mu+\frac{1}{2} s+\frac{1}{4}\right)}{\Gamma\left(\frac{1}{2} \mu-\frac{1}{2} s+\frac{3}{4}\right)},
\end{aligned}
$$

whence $q(x)=x^{\frac{1}{2}} J_{\mu}(x)$.

Thus, we have as a special case of Corollary 7 .

COROLLARY 8. If $f \in H_{v}$ and

$$
g(x)=\int_{0}^{\infty}\left(\frac{1}{2} x t\right)^{\frac{1}{2}(\mu+v+1)} K_{\frac{1}{2}(\nu-\mu)}(x t) f(t) d t,
$$


then $\quad g \in H_{\mu}$

Here the class $H_{\mu}$ denotes the class of self-reciprocal functions with respect to the Hankel transforms of order $\mu$. To varify this result, let

$$
f(x)=x^{v+\frac{1}{2}} e^{-\frac{1}{2} x^{2}},
$$

and $f(x) \in H_{\nu}$. Then, $[6 ; 132(25)]$,

$$
\begin{aligned}
g(x) & =\int_{0}^{\infty}\left(\frac{1}{2} x t\right)^{\frac{1}{2}(\mu+\nu+1)} K_{\frac{1}{2}(\nu-\mu)}(x t) f^{\prime}(t) d t \\
& =2^{\frac{1}{4}(\nu-\mu-4)} \Gamma(\nu+1) \Gamma\left(\frac{1}{2} \nu+\frac{1}{2} \mu+1\right) x^{\frac{1}{2}(\nu+\mu-1)} e^{x^{2 / 4} W_{-\frac{1}{4}}(\mu+3 \nu+2) ' \pm \frac{1}{4}(\nu-\mu)}\left(x^{2 / 2)}\right.
\end{aligned}
$$

and it is an easy matter to verify that $g(x) \in H_{\mu}$, as predicted, $[6 ; 84(15)]$. A particular case of the above result is obtained, if we set $\nu=\mu=\frac{1}{2}$, i.e.

COROLLARY 9. If $f \in R_{s}$ and

then

$$
g(x)=\int_{0}^{\infty} e^{-x t} f(t) d t
$$

$$
g(x) \in R_{c},
$$

where $R_{s}$ and $R_{c}$ denote the classes of self-reciprocal functions with respect to Fourier sine and Fourier cosine transforms, respectively. In the operational notation, we have that if

$$
F_{s}[f]=f \text { and } L[f]=g \text {, }
$$

then

$$
F_{c}[g]=g
$$

where $F_{S}, F_{C}$ and $L$ denote the Fourier sine, Fourier cosine and the Laplace transforms respectively. For example, consider

such that

$$
f(x)=\frac{2^{3 \nu+\frac{1}{2}}}{\Gamma(4 v+1)} x^{2 \nu-1} e^{-x^{2} / 4} M_{3 \nu, v}\left(x^{2} / 2\right), \text { Re } v>-\frac{1}{4},
$$

$$
\begin{aligned}
f(x) & \in R_{s},[7 ; 115(5)] . \\
\text { Now, } & \\
g(x) & =\int_{0}^{\infty} e^{-x t} f(t) d t \\
& =2^{-\nu} x^{2 \nu-1} e^{x^{2} / 4} W_{-3 \nu,-v}\left(x^{2} / 2\right),
\end{aligned}
$$

[7;215(13)], where $M_{\mu, \nu}$ and $W_{\mu, \nu}$ are Whittaker's functions and then $g(x) \in R_{c},[7 ; 61(7)]$, as predicted.

\section{REFERENCES}

1. E.C. Titchmarsh, Fourier Integrals, Clarendon Press, Oxford, 1948.

2. C. Fox, Chain transforms, J. London Math. Soc., 23 (1948), 229-235.

3. B.P. Duggal, On Fourier transform type operators, Period. Math. Hungary, 9 (1978), 55-61.

4. D.V. Widder, An Introduction to Transform Theory, Academic Press, New York, 1971.

5. C. Nasim, An inversion formula for a class of integral transforms, J. Math. Anal. App1. 52 (1975), 525-537.

6. A. Erdelyi, et al., Tables of Integral Transforms II, McGraw-Hill, New York, 1953.

7. A. Erdelyi, et al., Tables of Integral Transforms I, McGraw-Hill, New York, 1953. 


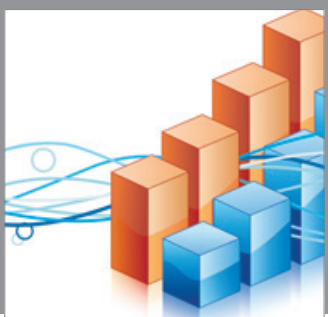

Advances in

Operations Research

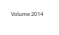

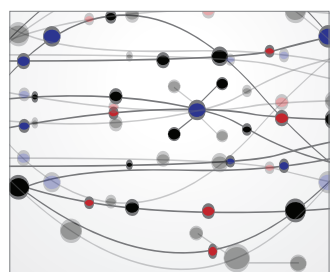

\section{The Scientific} World Journal
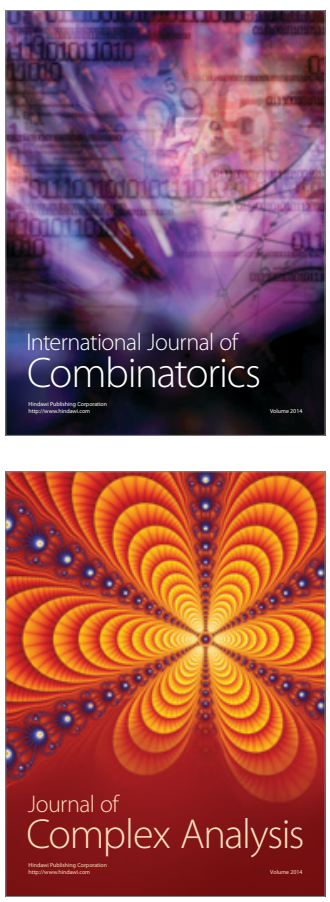

International Journal of

Mathematics and

Mathematical

Sciences
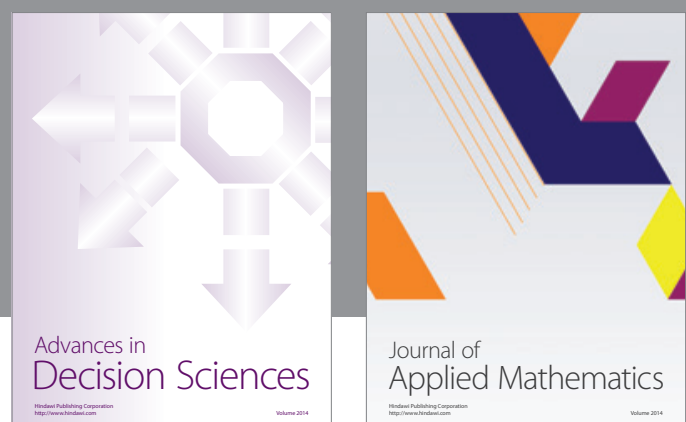

Journal of

Applied Mathematics
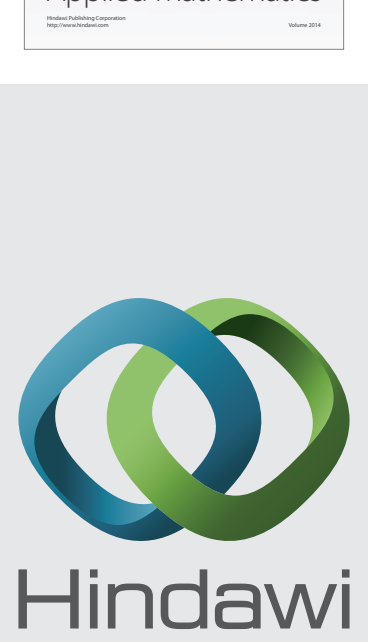

Submit your manuscripts at http://www.hindawi.com
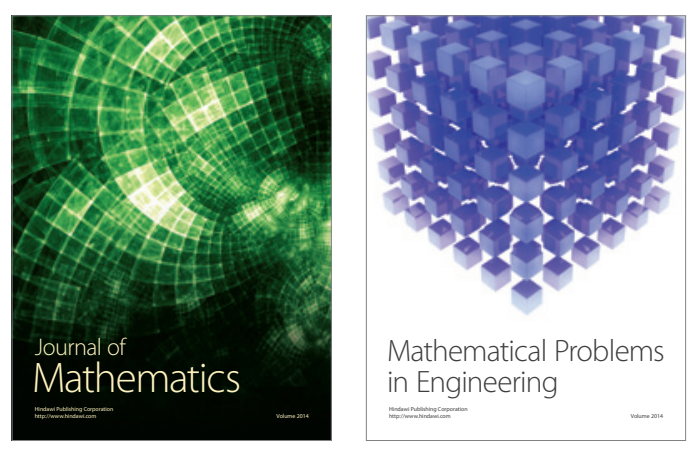

Mathematical Problems in Engineering
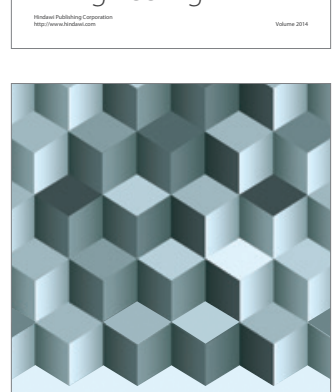

Journal of

Function Spaces
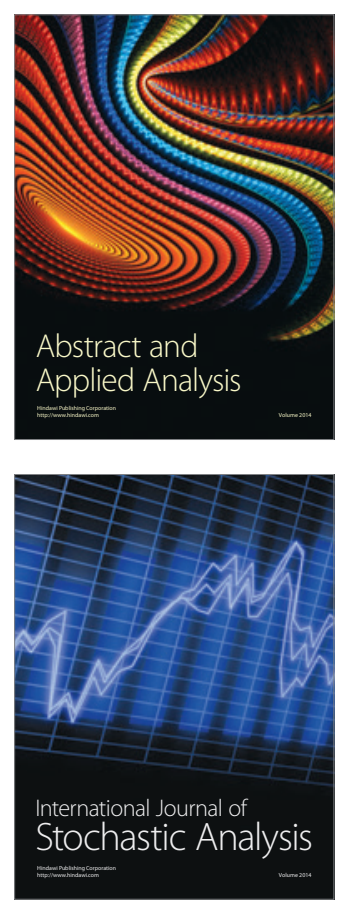

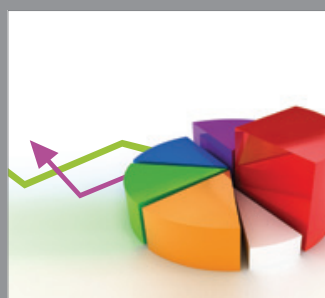

ournal of

Probability and Statistics

Promensencen
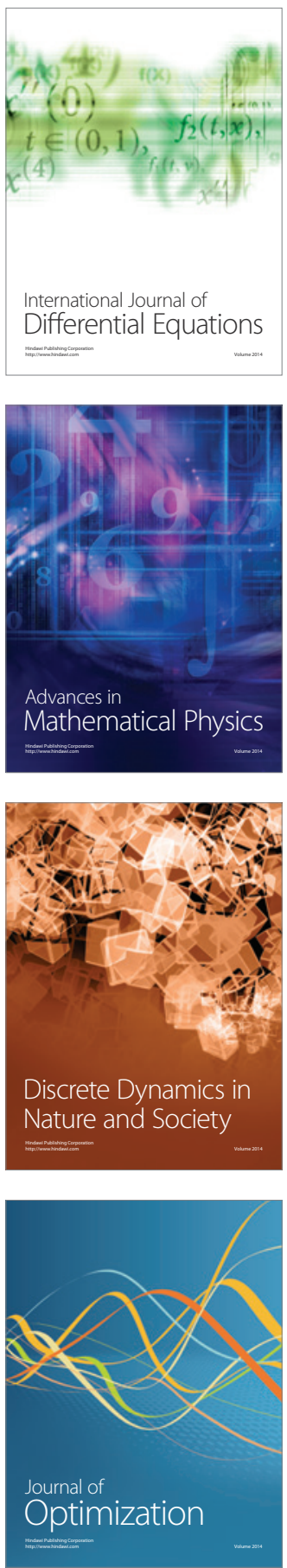